\title{
Relación entre la acumulación de días grado y el vuelo estacional de la mariposa europea del brote del pino en Esquel, Argentina*
}

The relationship between accumulated degree-days and the seasonal flight of the European pine shoot moth at Esquel, Argentina

\section{CECILIA ANDREA GOMEZ}

CONICET y Centro de Investigación y Extensión Forestal Andino Patagónico (CIEFAP). C.C. 14 (9200) Esquel. Chubut. E-mail: gomezcarabe@ar.inter.net

\section{SUMMARY}

The relationship between accumulated degree-days (DD) and the emergence of males of the European pine shoot moth (Rhyacionia buoliana Den. \& Schiff.) was determined using pheromone traps in a population of this species in a Pinus radiata plantation near Esquel City, Chubut province, at the Patagonian Andes region of Argentina. Traps were checked twice a week during three summer seasons from December 1999 to February 2002. On the second sampling season (2000 to 2001), 100 small branches with infected shoots were collected twice a week to estimate male and female emergence curves. Degree-days requirements (above $-2.2{ }^{\circ} \mathrm{C}$ ) for the seasonal flight of the males of this species were estimated. Predicted DD requirements were 252.26 DD for a $40 \%$ catch, 288.22 DD for a $50 \%$ catch and 434.53 DD for an $80 \%$ catch.

Key words: Rhyacionia buoliana, degree-days, seasonal flight, male catches.

\section{RESUMEN}

Se estudió la relación entre la acumulación de días grado (DG) y las capturas de los machos de la mariposa europea del brote del pino (Rhyacionia buoliana Den. \& Schiff.) en trampas de feromonas en Esquel, provincia de Chubut, en la zona andino-patagónica de Argentina, como un indicador para el control de esta especie plaga. En una plantación de Pinus radiata se monitoreó la emergencia de machos dos veces por semana mediante el recuento de las capturas en las trampas, durante tres temporadas (diciembre a febrero de 1999-2000, 2000-2001 y 2001- 2002). En la temporada 2000-2001 se recolectaron también dos veces por semana 100 ramillas con brotes infestados para construir la curva de emergencias de machos y de hembras. Los DG se calcularon en base a las temperaturas mínima y máxima diarias. Se estimaron los requerimientos de DG acumulados (por encima de $-2,2{ }^{\circ} \mathrm{C}$ ) para el período de vuelo del insecto. Los DG acumulados pronosticados por el modelo de regresión sigmoídea fueron 252,26 DG, 288,22 DG y 434,53 DG para el 40, 50 y $80 \%$ de las capturas de machos.

Palabras claves: Rhyacionia buoliana, días grado, vuelo estacional, capturas de machos.

\section{INTRODUCCION}

La mariposa europea del brote del pino (Rhyacionia buoliana Den. \& Schiff.) (Tortricidae) es una plaga específica del género Pinus, cuyas larvas se alimentan de los tejidos de las yemas y brotes provocando deformaciones en el fuste y ramas. En verano, la hembra deposita sus huevos preferentemente en los brotes nuevos, las larvas de los estadios uno y dos se alimentan de la base

\footnotetext{
* A la profesora Dolly Lanfranco de la Universidad Austral de Chile por la identificación de los parasitoides. Proyecto financiado por la International Foundation for Science (IFS), D/2874-1.
} 
de las acículas y luego el segundo estadio larval penetra en las yemas nuevas y se alimenta en su interior. Cuando la yema ha sido parcial o totalmente ahuecada, la larva se dirige a otra yema y continúa alimentándose hasta el invierno, en que entra en dormancia como larva del estadio tres y/o cuatro. Cuando la temperatura asciende a $15^{\circ} \mathrm{C}$ o más durante tres o cuatro días consecutivos las larvas reanudan su actividad; migran hacia la parte alta de las copas y continúan alimentándose en el interior de las yemas hasta alcanzar el último (sexto) estadio. La pupación ocurre en el interior de la yema aproximadamente durante octubre-noviembre y la emergencia de los adultos se inicia a principios de diciembre y continúa hasta comienzos de febrero dependiendo siempre de las condiciones climáticas.

En la región andino-patagónica de Argentina la plaga se comporta como univoltina y está presente desde 1979 afectando las plantaciones de diferentes especies de pinos con distinta intensidad de ataque.

Las plantaciones que corren mayor riesgo son aquellas que tienen entre tres y 12 años de edad (Robredo 1970). En lugares para recreación, los ataques reducen el valor estético, ya que en las plantas más grandes se destacan brotes laterales curvados, que son frecuentemente quebrados por el viento. Con el incremento de la superficie forestada en la última década, principalmente en las provincias de Neuquén y Chubut (Carabelli et al. 2000), se produjeron incrementos poblacionales de la mariposa del brote. Con ello aumenta el riesgo de pérdidas económicas, tal como ha sucedido en las plantaciones de Chile, predominantemente constituidas por Pinus radiata D. Don (Baldini et al. 1993, Baldini \& Alvarez de Araya 1998, Ide \& Lanfranco 1994).

El control de esta plaga mediante la aplicación de productos químicos se lleva a cabo cuando la intensidad de infestación es muy alta y se desea disminuir drásticamente la densidad poblacional para aplicar otro método de control más efectivo a largo plazo -tal como el control biológico-, o bien en el caso de los plantines en viveros. Dado que el insecto permanece protegido dentro del brote la mayor parte de su ciclo de vida, la aplicación de insecticidas se efectúa principalmente sobre los adultos y también sobre las larvas de los estadios uno y dos, que son los que permanecen más expuestos fuera de los brotes. La emergencia de los adultos puede predecirse mediante diferentes procedimientos: a) monitoreo de las pupas, método que asume que el primer vuelo ocurrirá cuando se alcance el $80 \%$ de pupas y el 20\% de larvas (Regan et al. 1991); b) construcción de las curvas de emergencia monitoreando los estados de desarrollo presentes y las exuvias de pupas; y c) cálculo de días grado (DG). Los métodos a y b requieren mucho trabajo y personal entrenado, además de un alto grado de infestación en la plantación. Ambos procedimientos resultan poco prácticos dadas las características de las plantaciones de la zona andino-patagónica de Argentina, donde el monitoreo está a cargo de personal de campo que fundamentalmente se ocupa de la vigilancia, sin entrenamiento para el relevamiento ni el reconocimiento de problemas fitosanitarios.

El método del cálculo de los días grado constituye una herramienta práctica, de fácil elaboración, y con la que el personal del lugar puede familiarizarse rápidamente. Consiste en llevar el registro diario de temperaturas y luego transformarlas a DG, para relacionarlas con las fechas del vuelo estacional de los adultos machos. Si se obtiene una relación estadísticamente significativa se puede utilizar para aumentar la eficiencia del control químico. La utilidad de los modelos DG ha sido demostrada para varias especies, entre otras Choristoneura fumiferana Clem. (Bean \& Wilson 1964), Eucosma sonomana Kearfott (Mitchell \& Sower 1991), y para $R$. buoliana en el oeste de Oregon, USA (Regan et al. 1991).

Los objetivos del presente trabajo son determinar la relación entre la acumulación de DG y el vuelo de los machos adultos como una herramienta en el manejo de esta plaga, y para contribuir al conocimiento del ciclo de vida de la mariposa europea del brote en la zona andino-patagónica de Argentina. Cabe destacar que es la primera vez que se realiza un estudio de esta naturaleza en la región.

\section{MATERIAL Y METODOS}

El estudio incluyó tres temporadas (6 semanas entre el 03 de diciembre de 1999 y el 10 de enero de 2000, 8 semanas entre el 17 de diciembre de 2000 y el 12 de febrero de 2001 y 7 semanas entre el 05 de diciembre de 2001 y el 24 de enero de 2002), durante las que se monitoreó el vuelo de 
machos adultos de la mariposa europea del brote del pino mediante su captura en trampas. El muestreo se llevó a cabo en una plantación de $P$. radiata de 14 años de edad y de una superficie aproximada de 3,5 ha ubicada al norte de la localidad de Esquel, en la provincia de Chubut. Se emplearon seis trampas delta con feromonas de tipo Biolure (EPSM Lures, Point International Ltd., Reino Unido) durante las dos primeras temporadas, y diez trampas durante la última temporada. Las mismas se dispusieron en grupos conformando rectángulos de dos filas y tres hileras en los que las trampas estaban distanciadas 25-30 m entre sí (Regan et al. 1991). Las trampas se colgaron de las plantas un mes o quince días antes que comenzara el período de vuelo (comienzos-mediados de noviembre), a una altura aproximada de 1,5-2 m, y se controlaron dos veces por semana hasta el momento en que no se capturaron más adultos. La frecuencia de control de las trampas durante la primera temporada fue menos intensa y sistemática, ya que se efectuaron sólo siete muestreos, mientras que durante la segunda temporada se efectuaron 16 muestreos y 13 muestreos durante la última temporada. Los cebos fueron reemplazados una vez durante el período de muestreo.

Con fines comparativos se construyó para la temporada 2000- 2001 la curva de emergencias de adultos de ambos sexos empleando el método del conteo de los estados de desarrollo presentes y de las exuvias de las pupas. Con la misma frecuencia que para la captura de machos, se recolectaron en cada ocasión de muestreo y en forma aleatoria aproximadamente 100 ramas con brotes infestados ( \pm 10 ramas) que se diseccionaron en el laboratorio para registrar el número de estados /estadios larvales y de exuvias en los brotes. Se colectaron no más de tres brotes por planta tratando de extraerlos de distintas partes de las mismas (estratos superior, medio e inferior y diferente exposición). Las larvas se diseccionaron en búsqueda de parasitoides, que se identificaron tanto por las larvas (Juillet 1960) como por los adultos que se obtuvieron en laboratorio luego de haber sido mantenidos en cajas plásticas hasta su emergencia. Este muestreo se realizó hasta registrar solamente exuvias en los brotes examinados. El porcentaje de emergencia se calculó empleando la siguiente fórmula (según Palma Prieto 1995):
Número de exuvias en $t$

Número total de individuos (larvas, pupas y exuvias)

$t=$ día de muestreo.

Cabe aclarar que en el denominador, las larvas que participan en la suma son sólo aquellas no parasitadas, puesto que las parasitadas no alcanzan el estado de pupa.

La temperatura se registró con un termohigrógrafo que, ante la eventualidad de que pudiera ser dañado, se instaló a $500 \mathrm{~m}$ de la plantación en un lugar más seguro y con vigilancia. El mismo registra la temperatura en forma continua desde su instalación en diciembre de 1999. Las temperaturas registradas se convirtieron en días grado empleando el método de Arnold (1959), que también se conoce como método simple, del rectángulo, de los máximos y mínimos, y es uno de los más comúnmente usados (Higley et al. 1986). La ecuación que emplea el método es la siguiente:

$$
D G=\frac{T_{\max }+T_{\min }}{2}-T_{\text {umbral }}
$$

T máx. = Temperatura máxima diaria $\left({ }^{\circ} \mathrm{C}\right)$

$\mathrm{T}$ mín. $=$ Temperatura mínima diaria $\left({ }^{\circ} \mathrm{C}\right)$

$\mathrm{T}$ umbral $=$ Temperatura umbral inferior $\left({ }^{\circ} \mathrm{C}\right)$, por debajo de la cual no hay desarrollo del organismo.

La fecha en que se registró la primera captura en las trampas se tomó como momento de inicio para el cálculo de DG. La temperatura umbral inferior considerada fue de $-2,2{ }^{\circ} \mathrm{C}$ (Regan et al. 1991). No se contempló un umbral superior pues en la región andino-patagónica las temperaturas raramente exceden los $35^{\circ} \mathrm{C}$.

Las capturas de machos de cada fecha de control de trampas fueron acumuladas y se calculó el porcentaje de capturas acumuladas en cada control respecto del total de capturas de todo el período de muestreo. Se construyó un modelo de regresión sigmoidea con los datos de las tres temporadas, adoptándose la función que brindó el mejor ajuste respecto de la distribución de los valores muestrales de temperatura y días grado. 
BOSQUE 24(3): 57-63, 2003

Relación entre la acumulación de días grado y el vuelo estacional de la mariposa europea del brote...

\section{RESULTADOS Y DISCUSION}

Los primeros machos se capturaron el 03/12 en la temporada 1999-2000, el 17/12 en la temporada 2000-2001 y el 5/12 en la temporada 2001-2002, mientras que las últimas capturas se registraron el $10 / 01$, el 8/02 y 21/01 para la primera, segunda y tercera temporada respectivamente (cuadro 1). La cantidad de machos capturados fue similar para la primera y la última temporada de muestreo -1.227 y 1.170 respectivamente-, sin embargo, durante la segunda temporada el número de machos capturados fue muy inferior (139). Esta diferencia podría explicarse por los fuertes vientos y lluvias que predominaron durante el período, disminuyendo drásticamente y/o dificultando el vuelo durante la segunda temporada. Según Green (1962) el vuelo de la mariposa del brote disminuye significativamente por debajo de los $11-12^{\circ} \mathrm{C}$, en tanto la lluvia tiene un efecto variable en el vuelo, dependiendo de su intensidad. Generalmente, cuanto más intensa es la lluvia menor es el vuelo.
El modelo de regresión sigmoidea para el porcentaje de captura acumulado y los DG mostró que ambas variables están bien correlacionadas; el modelo tiene la siguiente expresión: $y=\frac{a b+c x^{d}}{b+x^{d}}$ $\left(\mathrm{r}^{2}=0,982 ; \mathrm{s}=5,539\right.$ donde: $\mathrm{y}$ es el porcentaje de captura acumulado, $\mathrm{x}$ son los DG acumulados por encima de $-2,2{ }^{\circ} \mathrm{C}$ y los valores de los parámetros del modelo: $\mathrm{a}=1,2522199, \mathrm{~b}=16196888$, $\mathrm{c}=109,02445 \mathrm{y} \mathrm{d}=2,8972109$ ).

El requerimiento de temperatura pronosticado según el modelo para el 30,40 y $80 \%$ de vuelo resultó de 217,18 DG, 252,26 DG y 434, 53 DG respectivamente (figura 1). Los porcentajes acumulados de capturas de machos son valores claves en el manejo de esta plaga. La eficiencia de los insecticidas está relacionada con el porcentaje de emergencia de los adultos y de eclosión de los huevos (Butcher \& Haynes 1960). Asimismo, la aplicación de los productos químicos se realiza cuando se registra la emergencia del $30-40 \%$ de adultos de ambos sexos o bien del $80 \%$, dependiendo del tipo de compuesto que se aplique (Goycoolea, com. pers.).

\section{CUADRO 1}

Detalle de los DG acumulados y porcentaje de captura acumulada en cada fecha de muestreo y para cada temporada.

Accumulated DD and percentage of catches for each sampling date and season.

\begin{tabular}{|c|r|r|r|r|r|r|r|r|}
\hline \multicolumn{2}{|c|}{ Temporada 1999-2000 } & \multicolumn{2}{|c|}{ Temporada 2000-2001 } & \multicolumn{3}{c|}{ Temporada 2001-2002 } \\
\hline \multirow{2}{*}{ Fecha } & \% capt. & DG acum. & Fecha & \% capt. & DG acum. & Fecha & \% capt. & DG acum. \\
\hline \multirow{2}{*}{$03 / 12 / 99$} & 4 & 9,20 & $17 / 12 / 00$ & 0,72 & 16,95 & $05 / 12 / 01$ & 0,17 & 16,35 \\
$06 / 12 / 99$ & 4,16 & 29,50 & $21 / 12 / 00$ & 5,75 & 72,90 & $10 / 12 / 01$ & 1,45 & 75,55 \\
$14 / 12 / 99$ & 25,92 & 180,00 & $25 / 12 / 00$ & 10,07 & 133,25 & $14 / 12 / 01$ & 13,99 & 148,10 \\
$21 / 12 / 99$ & 39,7 & 303,35 & $29 / 12 / 00$ & 10,79 & 182,55 & $21 / 12 / 01$ & 35,49 & 269,10 \\
$31 / 12 / 99$ & 73,02 & 464,00 & $02 / 01 / 01$ & 43,16 & 259,20 & $26 / 12 / 01$ & 62,80 & 352,35 \\
$03 / 01 / 00$ & 96,58 & 521,85 & $04 / 01 / 01$ & 65,47 & 304,45 & $29 / 12 / 01$ & 75,94 & 398,85 \\
$10 / 01 / 00$ & 100,00 & 627,35 & $08 / 01 / 01$ & 74,82 & 375,25 & $02 / 01 / 02$ & 90,96 & 470,50 \\
& & & $11 / 01 / 01$ & 76,26 & 410,65 & $05 / 01 / 02$ & 97,87 & 510,35 \\
& & & $15 / 01 / 01$ & 84,17 & 467,50 & $08 / 01 / 02$ & 98,46 & 558,05 \\
& & & $18 / 01 / 01$ & 88,49 & 512,10 & $12 / 01 / 02$ & 99,66 & 620,85 \\
& & & $22 / 01 / 01$ & 92,10 & 570,65 & $18 / 01 / 02$ & 99,83 & 723,50 \\
& & & $25 / 01 / 01$ & 94,96 & 621,15 & $21 / 01 / 02$ & 100,00 & 784,10 \\
& & & $29 / 01 / 01$ & 98,56 & 683,45 & $24 / 01 / 02$ & 100,00 & 841,70 \\
& & & $05 / 02 / 01$ & 99,28 & 786,10 & & & \\
& & & $08 / 02 / 01$ & 100,00 & 828,20 & & & \\
& & & $12 / 02 / 01$ & 100,00 & 881,10 & & \\
\end{tabular}




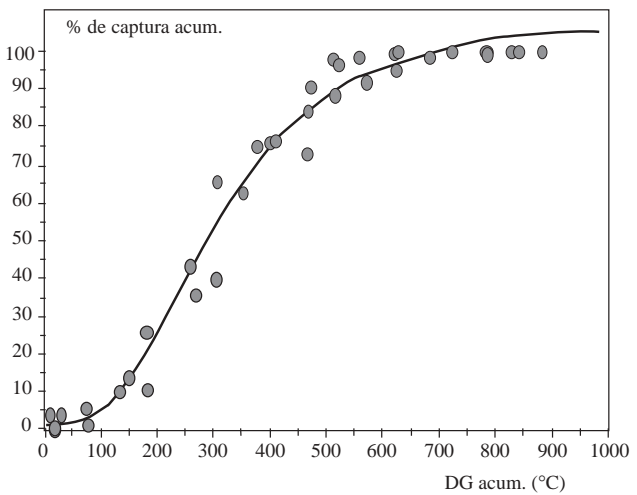

Figura 1. Capturas acumuladas de machos adultos en vuelo vs. DG acumulados (temporadas 1999-2000, 20002001 y 2001-2002).

Accumulated catches of males in flight versus the accumulated DD (flight seasons: 1999 to 2000, 2000 to 2001 and 2001 to 2002).

Las curvas de captura de machos y de emergencias de adultos de ambos sexos tendieron a ser sigmoideas (figuras 1 y 2), como se ha obtenido para otras especies como $R$. frustrana Comstock (Richmond et al. 1983). Sin embargo, se observan algunas oscilaciones en la curva de emergencias de adultos de ambos sexos, las que pueden atribuirse a la pérdida de exuvias por el viento o a la dificultad para detectarlas en ramillas muy atacadas. Además, hay que considerar que no emerge

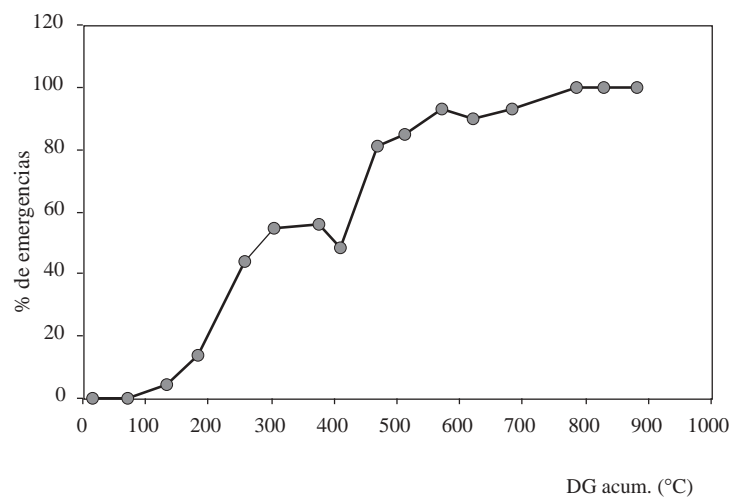

Figura 2. Porcentaje de emergencia de adultos de ambos sexos vs. DG acumulados, temporada 2000-2001.

Emergence percentage of adults of both sexes versus the accumulated DD in the 2000 to 2001 season.

el $100 \%$ de las pupas debido al parasitismo, predación, etc. Si bien las larvas parasitadas no se consideraron en el cálculo de los porcentajes de emergencia, se destaca que a partir de la captura del primer macho se extrajeron 43 larvas (22 pertenecientes al estadio cinco y 21 al estadio seis) de un total de 1.100 brotes disectados. El $67 \%$ de estas larvas estaban parasitadas con el bracónido Orgilus obscurator Ness, por lo que no alcanzarían el estado de pupa (cuadro 2).

\section{CUADRO 2}

Muestreo de exuvias y porcentaje de emergencia de adultos Exuvia sampling and percentage of adult emergence.

\begin{tabular}{|c|r|c|r|r|r|r|r|r|}
\multirow{2}{*}{ Fecha } & \multicolumn{3}{|c|}{$N^{\circ}$ larvas } & \multicolumn{3}{c|}{$N^{\circ}$ pupas } & \multirow{2}{*}{$N^{\circ}$ exuvias } & \% emerg. \\
\cline { 2 - 7 } & Parasit. & No parasit. & Total & Machos & Hembras & Total & & \\
\hline 17/12/00 & 0 & 8 & 8 & 47 & 37 & 84 & 0 & 0 \\
$21 / 12 / 00$ & 17 & 5 & 22 & 62 & 59 & 124 & 0 & 0 \\
$25 / 12 / 00$ & 4 & 0 & 4 & 94 & 81 & 175 & 8 & 4 \\
$29 / 12 / 00$ & 1 & 1 & 2 & 56 & 53 & 109 & 18 & 14 \\
$02 / 01 / 01$ & 1 & 0 & 1 & 20 & 29 & 49 & 39 & 44 \\
$04 / 01 / 01$ & 0 & 0 & 0 & 25 & 15 & 40 & 49 & 55 \\
$08 / 01 / 01$ & 0 & 0 & 0 & 10 & 21 & 31 & 40 & 56 \\
$11 / 01 / 01$ & 0 & 0 & 0 & 13 & 22 & 35 & 34 & 49 \\
$15 / 01 / 01$ & 5 & 0 & 5 & 2 & 3 & 5 & 42 & 81 \\
$18 / 01 / 01$ & 0 & 0 & 0 & 1 & 8 & 9 & 53 & 85 \\
$22 / 01 / 01$ & 0 & 0 & 0 & 0 & 4 & 4 & 53 & 93 \\
$25 / 01 / 01$ & 0 & 0 & 0 & 2 & 3 & 5 & 44 & 90 \\
$29 / 01 / 01$ & 0 & 1 & 1 & 2 & 1 & 3 & 51 & 93 \\
$05 / 02 / 01$ & 0 & 0 & 0 & 0 & 0 & 0 & 33 & 100 \\
$08 / 02 / 01$ & 0 & 0 & 0 & 0 & 0 & 0 & 35 & 100 \\
\hline
\end{tabular}


BOSQUE 24(3): 57-63, 2003

Relación entre la acumulación de días grado y el vuelo estacional de la mariposa europea del brote...

En la figura 3 se observa que en las curvas de capturas no acumuladas para las tres temporadas de muestreo aparece una mayor concentración de valores de porcentajes de captura en torno a un valor central de DG. Sin embargo, este valor central de DG no coincide para las tres temporadas, sino que hay un desplazamiento entre ellas indicando una diferencia de aproximadamente 100 y 200 DG. Se espera que en las próximas temporadas de muestreo aparezca una tendencia hacia una u otra curva. Una de las posibles explicaciones podría ser que se comenzó a registrar la acumulación de los DG a partir de la primera captura de un macho en vuelo; concretamente el 01/12 de 1999 para la primera, el $17 / 12$ para la segunda temporada y $05 / 12$ para la tercera. Ese retraso de aproximadamente unas dos semanas en la segunda temporada respecto de la primera y la tercera, sugiere que puede haber habido diferencias importantes en las temperaturas previas a las fechas de las primeras capturas de machos en vuelo. Sin embargo, este efecto se desecha puesto que la acumulación de temperaturas de dos semanas previas a la fecha de la primera captura fue de 248 DG y 171,7 DG para la segunda y para la tercera temporada, respectivamente. No se dispone de los registros previos a la captura correspondiente a la primera temporada de muestreo.

La trampa con feromonas capturó el primer macho ocho días antes de que fueran detectadas las primeras exuvias en los brotes.
En la zona de Los Arenales de la VIII Región chilena, un estudio similar basado en el conteo de exuvias arrojó los siguientes resultados (para dos estaciones climáticas diferentes y para un sólo período anual de muestreo): 241,5-184,2 DG para el $50 \%$ de emergencia y $298,1-270,7^{\circ} \mathrm{C}$ para el $80 \%$ de emergencia (Palma Prieto 1995). Estos valores difieren entre 47 y 104 DG para el 50\% de las capturas y 136 y 164 DG para el $80 \%$ de las capturas de los valores predichos por la función que ajusta la curva sigmoidea obtenida en el presente estudio (figura 1). Posiblemente, estas discrepancias se deban a diferencias climáticas a excepción de la temperatura, pues se supone que el desarrollo de una especie tiene los mismos requerimientos térmicos independientemente de la ubicación geográfica, a menos que haya diferencias de biotipos, por ejemplo. Por otro lado, el estudio realizado en Chile se hizo en base al registro de exuvias -que generalmente es más variable puesto que el número de exuvias halladas no indica necesariamente que haya salido el mismo número de adultos-, mientras que este trabajo presenta estimaciones basadas en la captura de machos con trampas.

Regan et al. (1991) realizaron un estudio similar basado en las capturas de machos. Según ese estudio se necesitan 1.712 DG, 1.958 DG y 2.205 DG para el $10 \%, 50 \%$ y $90 \%$ de las capturas en trampas, respectivamente. Sin embargo, esos resultados no pueden compararse con los obtenidos en este

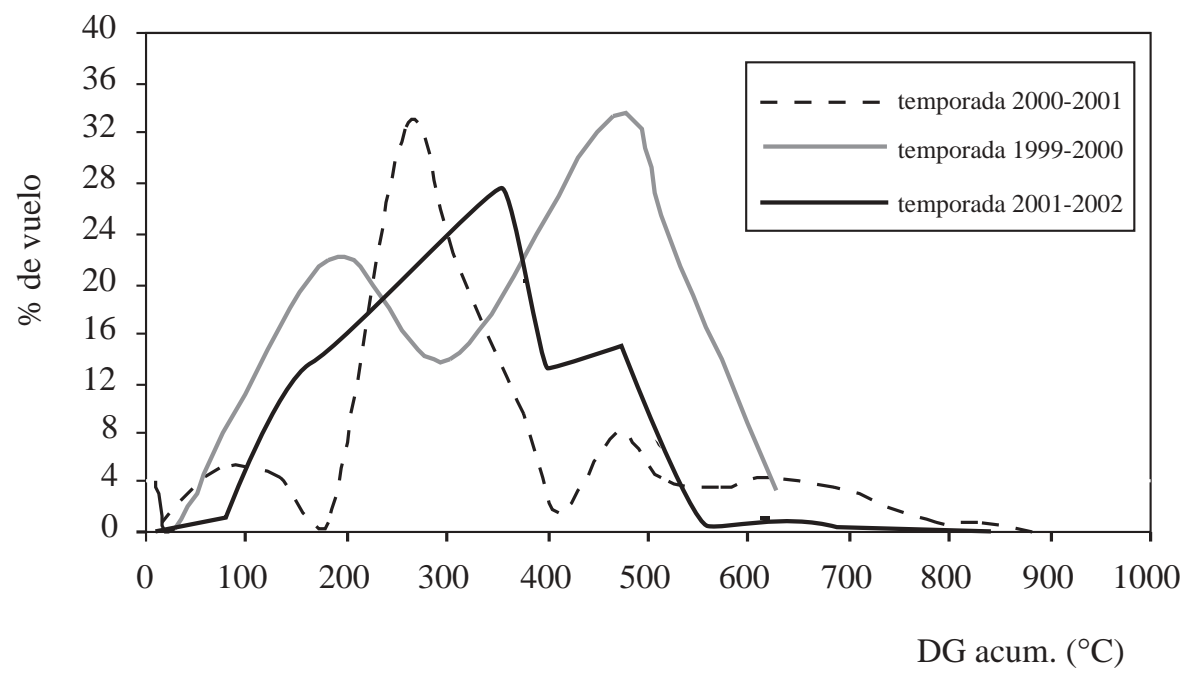

Figura 3. Capturas no acumuladas de machos adultos en vuelo vs. DG acumulados. Non-accumulated catches of males in flight versus the accumulated DD. 
estudio, puesto que no emplea un biofix sino una fecha fija para comenzar a acumular los DG.

Los resultados de este trabajo, aun teniendo en cuenta su carácter preliminar, constituyen los primeros elementos para la construcción de un modelo fenológico que debe ser ajustado en las próximas temporadas para examinar su capacidad de predicción.

\section{BIBLIOGRAFIA}

ARNOLD, C. 1959. "The determination and significance of the base temperature in a linear heat unit system", Soc. Proc. Am. Hortic. Sci. 74: 430-445.

BALDINI, A., G. ALVAREZ DE ARAYA, G. TORNQUIST, O. PUENTES. 1993. Evaluación de las pérdidas de volumen aprovechable ocasionadas por la polilla del brote en plantaciones de pino. CONAF, Departamento de Manejo y Desarrollo Forestal, Santiago, Chile. 22 p.

BALDINI, A., G. ALVAREZ DE ARAYA. 1998. "Evaluación de pérdidas económicas en plantaciones de Pinus radiata ocasionadas por polilla del brote". Actas del Congreso Latinoamericano de Plagas Forestales. IUFRO. Chile.

BEAN, L., F. WILSON. 1964. "Comparing various methods for predicting development of the spruce budworm, Choristoneura fumiferana, in northern Minnesota", J. Econ. Entomol. 57 (6): 925-928.

BUTCHER, J., D. HAYNES. 1960. "Influence of timing and insect biology on the effectiveness of insecticides applied for control of European pine shoot moth Rhyacionia buoliana". J. Econ. Entomol. 53: 349-354.

CARABELLI, F., S. ANTEQUERA, G. MARTIN, M. GOMEZ. 2000. Análisis Ambiental y Social de las Cuencas Hidrográficas Cordilleranas de la Provincia del Chubut.
CIEFAP, GTZ y Dirección General de Bosques y Parques, Serie Técnica $\mathrm{N}^{\circ} 5.56 \mathrm{p}$.

GREEN, W. 1962. "Flight and dispersal of the European pine shoot moth, Rhyacionia buoliana (Schiff.). I. Factors affecting flight, and the flight potential of females", Can. Ent. 94: 282-298.

HIGLEY, L., L. PEDIGO, K. OSTLIE. 1986. "Degday: A Program for calculating Degree-days, and assumptions behind the degree-day approach", Environ. Entomol. 15: 999-1.016.

IDE, S., D. LANFRANCO. 1994. "Fluctuaciones poblacionales de Rhyacionia buoliana (Lep.: Tortricidae) en una plantación de Pinus radiata en la X Región”, Bosque: 15 (2): $39-44$.

JUILLET, J. 1960. "Immature stage, life histories and behavior of two hymenopterous parasites of the European pine shoot moth, Rhyacionia buoliana (Schiff.) (Lepidoptera: Olethreutidae)", Can. Ent. 92: 342-346.

MITCHELL, G., L. SOWER. 1991. "Life history of the western pine shoot borer (Lepidoptera: Olethreutidae) and effects on lodgepole pine in Central Oregon", J. Econ. Entomol. 84 (1): 206-211.

PALMA PRIETO, S. 1995. Relación entre la acumulación de días grado y el ciclo biológico de la polilla del brote (Rhyacionia buoliana Den. et Schiff.) en la zona de Los Arenales, VIII Región. Memoria para optar al título de Ingeniero Forestal. Universidad de Chile, Santiago, Chile. $186 \mathrm{p}$.

REGAN, R., J. DE ANGELIS, G. GREDLER. 1991. "Predicting seasonal flight of European Pine Shoot Moth (Lepidoptera: Tortricidae) in Western Oregon", Ent. Soc. of Am. 20 (5): 1.403-1.406.

RICHMOND, J, H. THOMAS, H. BHATTACHARYYA. 1983. "Predicting spring flight of Nantucket pine tip moth (Lepidoptera: Olethreutidae) by heat unit accumulation", $J$. Econ. Entomol. 76: 269-271.

ROBREDO, F. 1970 "Contribución al conocimiento de la bioecología de Rhyacionia buoliana Schiff. y sus daños", Bol. Serv. de Plagas Forestales 13 (26): 181-186. 